\title{
Performance testing for high-speed train tracing and an approach-alarming system
}

\author{
J. Wang, B. Cai, J. Liu \& W. Shangguan \\ School of Electronics and Information Engineering, \\ Beijing Jiaotong University, China
}

\begin{abstract}
High-speed train tracing and an approach-alarming system is independent of the existing train control system, which uses the Global Navigation Satellite System and Integrated Location System for positioning to ensure the safety of trains and to prevent an accident of train collision caused by failure of the train control system. The system provides autonomous train tracing and positioning services, and sends different levels of safety alarm according to the distance between the adjacent trains. Based on the performance testing conducted on HanYi Railway of China (from Wuhan to YiChang), the requirements, architecture and working principle of the system, as well as the purpose, conditions, scene setting and process of the testing are expounded in this paper. According to three function indexes including the judgment of the train's position, analysis of the train's running mode and its transforming, plus the state of alarming and its transforming, the test data have been analyzed and discussed. At the end, the evaluation and conclusion of the testing are provided with a further discussion on the deficiency of and remedial measures for the system.
\end{abstract}

Keywords: high-speed train, tracing and approach-alarming, GPS, DGPS.

\section{Introduction}

Currently, the high-speed railway in China has developed and been applied rapidly. When it provides fast, convenient transportation service for people, its operation safety has been the key problem that restricts and affects the transportation of high-speed trains. WenZhou "7.23" rear-end accident has exposed the serious problems and deficiencies of existing signal system in highspeed train collision avoidance. The high-speed train control system has some 
inevitable failures in actual operation; therefore, the additional auxiliary collision alarming system as a supplement is needed to guarantee train operation safety.

High-speed train tracing and an approach-alarming system is independent of existing signal system using non-signal mode [1]. It integrates train positioning information and safety alarm logic, takes the advantage of multi-information transmission mode, and then efficiently calculates and evaluates safety state of the trains running on the same track and the same direction in real time. So it can provide a reasonable, accurate and efficient safety alarm for possible dangerous situations both in train operation and failure of signal system.

High-speed train tracing and approach-alarming system is designed to monitor and track the interval between trains. When the interval is below safe threshold and there could be a potential rear-end collision, the system can alarm the driver of the dangerous situation to take immediate actions to avoid accident. Germany demonstrated a train collision avoidance system on a test railway section in 2010; the Japanese Shinkansen also has a "train approach-alarming system" applied in operation.

Beijing Jiaotong University has been studying "the method of high-speed train tracing and approach-alarming system" and "the equipment of high-speed train tracing and approach-alarming system" since September 2011. Our team developed a prototype and then conducted a system testing on HanYi Railway of China on June $1^{\text {st }}-15^{\text {th }}$ 2012. We set different experimental conditions and scenarios to test system function, including train position judgment, state and transformation of alarming.

This paper firstly introduces the structure and key technology of high-speed train tracing and approach-alarming system, and then presents and analyzes the design, process and conclusion of the system testing.

\section{System structure and key technology}

High-speed train tracing and approach-alarming system is independent of the existing train control system, which uses the Global Navigation Satellite System and Integrated Location System for positioning to ensure the safety of trains and to prevent the accident of train collision caused by failure of the train control system.

\subsection{System structure and design principles}

High-speed train tracing and approach-alarming system consists of three parts: on-board device, ground equipment and wireless transmission device [2].

The on-board device is composed of on-board processing unit, alarm display device, train positioning unit, wheel detector and other devices. Ground equipment includes GPRS interface server for maintenance information and alarm server. Wireless transmission device is the railway dedicated GSM wireless communication device (GSM-R).

The system uses a GPS receiver and wheel detector for train positioning, and matches the on-board map database to acquire accurate train position, and then 
transmits the location information via train-ground wireless communication. The train alarm server makes comprehensive detection and processing of location information of each train in the area, and then conducts logic calculation of location judgment and dynamic distance calculation between front and back trains. Once the distance is below the predetermined safe limit, the safety alarm sever will send alarm information to the back train through GSM-R network.

The on-board device receives the alarm information, and presents current safety sate on display device in order to provide assist for driver's operation. The working principle of the system is shown in Fig. 1.

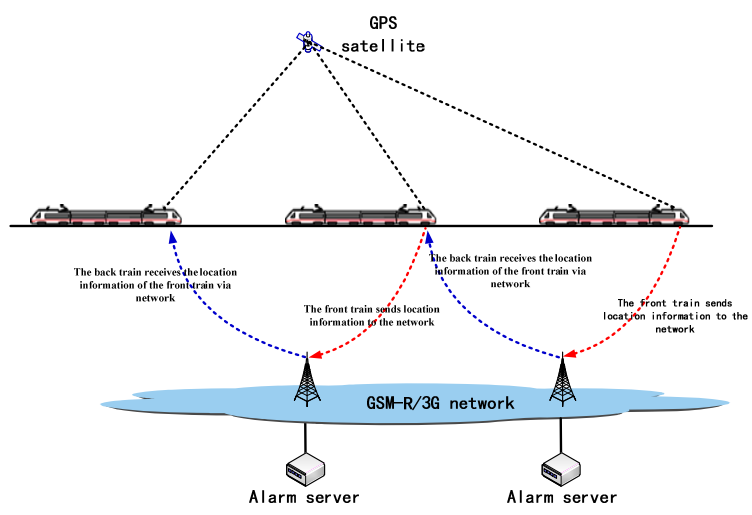

Figure 1: Technical principles.

The main technical principles of the system design are as follows:

- The system applies to passenger dedicated line and high-speed railway.

- The system applies to trains running on main tracks, and can also be applied to trains running on sidings.

- The system must be independent of the existing signal system, and doesn't use the data from the signal system.

- Train positioning is mainly based on satellite positioning with wheel detector, while wheel detector is used for assisted positioning in tunnel and GPS weak field.

- The system can send alarm information when the interval between the trains running on the same track and same direction is less than the safe threshold.

- The alarm is only sent to the driver, and let the driver decided what to do after receiving the message.

- The system needs to reduce the probability of false alarm as far as possible to avoid interference for the driver.

\subsection{Integrated positioning technology in high-speed train}

High-speed train tracing and approach-alarming system integrates train positioning information and safety alarm logic, takes the advantage of multi- 
information transmission mode, and then efficiently calculates and evaluates safety state of the trains running on the same track and the same direction in real time.

\subsubsection{Calculation logic of train positioning}

High-speed train tracing and approach-alarming system uses on-board device to positioning automatically. The position calculation adopts a completely independent configuration of hardware and software and calculation process, which doesn't rely on any shared device to acquire original input.

The on-board device includes one or two sets of GPS satellite positioning receiver and wheel detector, and it adopts comprehensive decision logic to determine train position. The initial result of the sensor is $2 \mathrm{D}$ coordinate position (longitude and latitude). By matching the result with the database of digital track, the kilometer marker as the $1 \mathrm{D}$ position property will be obtained. The onedimensional position represents the distance that the train travels from start station on track. On-board device can give available and continuous outputs in the condition of full situation (i.e., the sensors are working properly, and satellite visibility requirements are met) and non-full situation (sensors have faults, or the number of visible satellites is less than calculating requirement). The testing uses a single set of independent logic computation to positioning. The information processing flow is shown in Fig. 2.

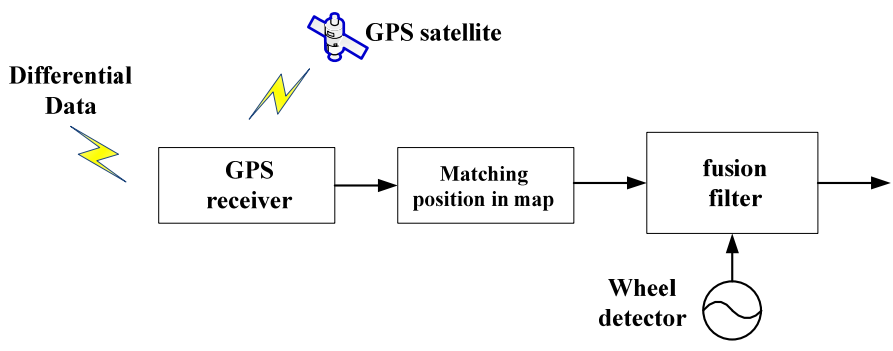

Figure 2: Information processing flow.

\subsubsection{Continuous train positioning in railway sections}

Errors exist in GPS positioning, so the anchor points acquired directly are discrete irregular points for map database, which means they cannot accurately represent actual train position. Map-matching algorithm [3] is designed to match the anchor points with the data points in map database in order to reckon the corresponding points on track.

Weight-based map-matching scheme mainly takes the following weights into consideration.

(1) The distance between anchor point and matching point of track in map database. Calculate the minimum distance with each candidate track, and the weight of the minimum distance $r$ is $w_{r}=A_{r}(1-r / d)$, in which $A r$ denotes weight coefficient, $d$ represents error threshold. From the formula, it can be seen: 
when the distance $r$ is getting larger, the weight is becoming smaller. And When $r$ is bigger than $d$, the weight is 0 .

(2) The number of previous matching track. According to the topology structure of track, before the train encounters the switch key point, the train can't leave current track. Its weight is $w_{n}=A_{n} a$ and when the train doesn't encounter the switch point, $a$ equals 1 ; when it does, $a$ equals 0 . In other words, after encountering the switch point, the matching result at the moment is not affected by the influence of previous matching result. When the train encounters the switch key point of start signal, the matching point should be default set back to the main track that connects the siding.

(3) The determination of train's running direction. High-speed railway track is usually divided into up line and down line. The matching track can be judged based on the train's running direction whose weight is $w_{d}=A_{d} d$. When the direction is as same as up and down line, $d$ is 1 , otherwise it's 0 .

(4) Train's history running record. Train operation plan will not change under non-special circumstances, so the moving trajectory of the train can be predicted according to history running record, especially in switch section. And its weight is $w_{h}=A_{h} h / H$, where $h$ denotes the historical statistics of track occupation, and $H$ denotes the total statistics.

(5) The number of GPS satellites. The number of satellites that GPS receiver can receive determines the accuracy of positioning, so it is included in the matching algorithm as a judgment factor. The receiver can receive 12 satellites at most, so when the number is $n$, its weight is $w_{s}=A_{s} n / 12$.

The weight formula of the train relating to each candidate track can be calculated as:

$$
\begin{gathered}
w=w_{r}+w_{n}+w_{d}+w_{h}+w_{s} \\
w=A_{r}(1-r / d)+A_{n} a+A_{d} d+A_{h} h / H+A_{s} n / 12
\end{gathered}
$$

in which, $A_{r}+A_{n}+A_{d}+A_{h}+A_{s}=1$. Determination of weight coefficient has great influence on the matching result; therefore GPS positioning performance, track topology and other information need to be considered to determine the weight coefficients of each part. In general, the parameters can be optimized through lots of experiments.

In a railway section, there are many scenes of GPS not positioning or too large positioning error, so additional corrections are needed. On-board device calculates final position of the train on track by fusing matching data and pulse data of velocity sensor together. Positioning train on track mainly relies on kilometer marker, namely one-dimensional data updating mode. The updating flow is shown in Fig. 3.

\subsubsection{Track occupancy identification and positioning at station}

When the train runs at railway station, the state of track occupancy can be identified without the aid of any signal system, so a fully autonomous 
recognition method can be adopted $[4,5]$. It integrates the satellite positioning data and inertia sensor data together to identify the track train runs on in order to positioning train at station. The identification process is shown in Fig. 4.

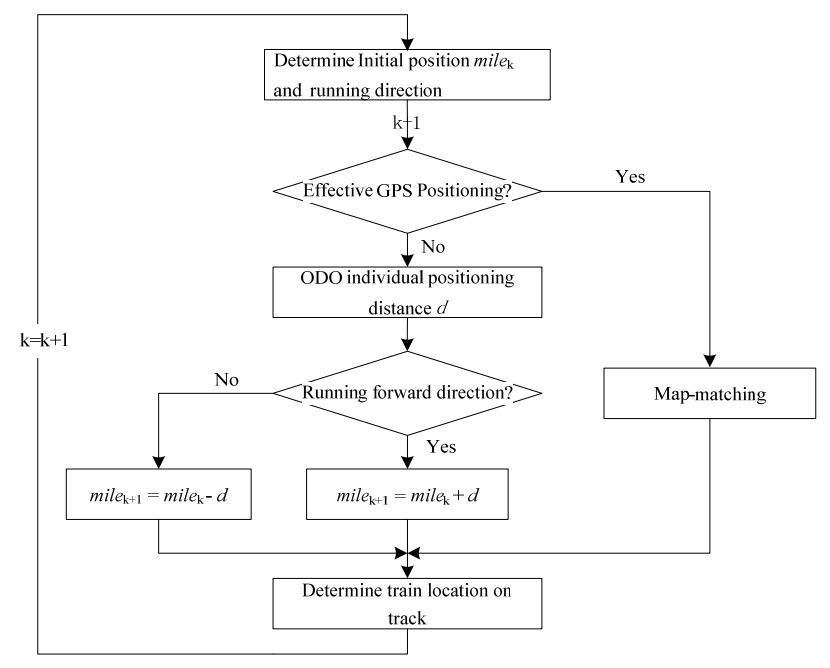

Figure 3: Information fusion process.

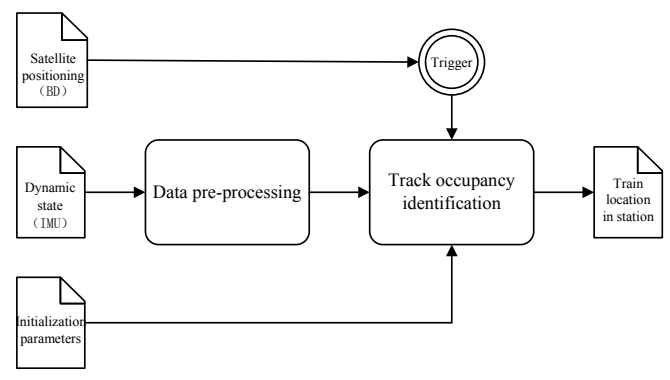

Figure 4: Process of track occupancy identification.

The identification is on the base of train route and track form, and it adopts syntactic analysis pattern recognition method, which extracts primitives of heading angle changes of train passing switches to model (shown in Fig. 5) [6].

Then it builds corresponding syntax based on operating mode (straight forward $L\left(G_{F}\right)$, turn into left line $L\left(G_{L}\right)$, and turn into right line $L\left(G_{R}\right)$ ), and determines the running line so as to deduce the track that the train occupies. Each syntax can be presented by a quaternion and set as follows: 


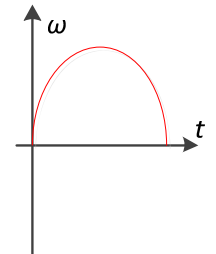

Left- turn- $l$

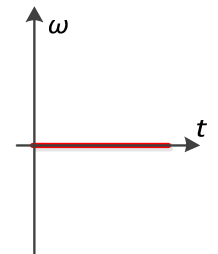

Straight $-f$

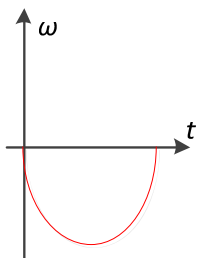

Right-turn - $r$

Figure 5: Method of track occupancy identification.

$$
\left\{\begin{array}{l}
G_{F}=\left\{V_{N F}, V_{T}, P_{F}, S\right\} \\
G_{L}=\left\{V_{N L}, V_{T}, P_{L}, S\right\} \\
G_{R}=\left\{V_{N R}, V_{T}, P_{R}, S\right\}
\end{array}\right.
$$

where non-terminators $V_{N F}=\{S, A\}, \quad V_{N L}=\{S, A, B, C, D, E\} \quad$ and $V_{N R}=\{S, A, H, I, J, K\}$ denote the sets of sub-mode and sentence of primitives; terminator $V_{T}=\{l, f, r, t\}$ is the set of the simplest primitives; production represents the relationship between terminator and non-terminators:

$$
\begin{aligned}
& P_{F}: S \rightarrow f A, A \rightarrow f A, A \rightarrow t ; \\
& P_{L}: S \rightarrow f A, A \rightarrow f A, A \rightarrow l B, B \rightarrow l B, B \rightarrow f C, B \rightarrow r D, \\
B \rightarrow & t, C \rightarrow f C, C \rightarrow r D, C \rightarrow t, D \rightarrow r D, D \rightarrow f E, D \rightarrow t, \\
E \rightarrow & f E, E \rightarrow t ; \\
& P_{R}: S \rightarrow f A, A \rightarrow f A, A \rightarrow r H, H \rightarrow r H, H \rightarrow f I, H \rightarrow l J, \\
H \rightarrow & t, I \rightarrow f I, I \rightarrow l J, I \rightarrow t, J \rightarrow l J, J \rightarrow f K, J \rightarrow t, K \rightarrow f K, \\
K \rightarrow & t .
\end{aligned}
$$

The identification uses finite state automaton, and its input-output relation is shown is Fig. 6.

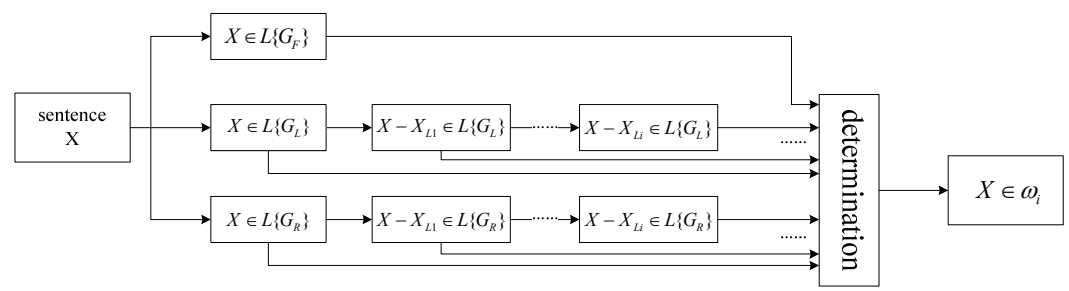

Figure 6: Process of pattern recognition. 


\section{Testing design}

On June 1-15 2012, the system development team conducted a testing of highspeed train tracing and the approach-alarming system in HanYi Railway.

\subsection{Testing conditions}

HanYi Railway is a part of Huhanrong Passenger Dedicated Line, from HanKou Station in the east to YiChang East Station in the west, passing Hanchuan, Xiantao, Tianmen, Qianjiang, Jingzhou, Zhijiang and other cities. It is 293.1 kilometers in length, and there are 8 stations and 1 line station (Wujiashan line station). The main technical standards are I -grade double-track electrified railway with target speed at $200 \mathrm{~km} / \mathrm{h}$, straight line spacing $4.6 \mathrm{~m}$, main line spacing $5.0 \mathrm{~m}$ at station, minimum curve radius $5495 \mathrm{~m}$ in railway section, effective length of arrival-departure track $850 \mathrm{~m}$. And the communication system is based on GSM-R.

The field testing is mainly done based on "the testing program of high-speed train tracing and an approach-alarming system". One set of center device is built on the ground, two outfits are set in differential base stations and four sets of onboard device are mounted on each front and tail of two trains. The testing equipment is shown in Fig. 7.

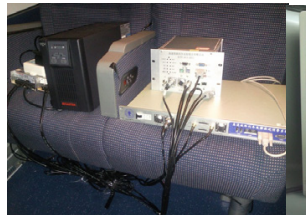

(a)

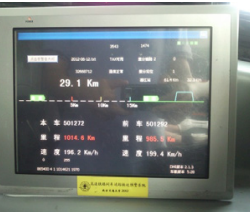

(b)

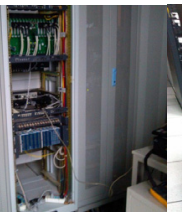

(c)

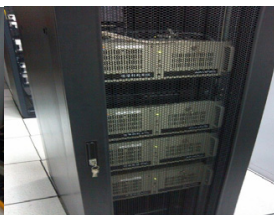

(d)

Figure 7: Testing equipments in field: (a) on-board alarm unit; (b) alarm display device; (c) differential base station; (d) center server on the ground.

\subsection{Testing project}

In terms of the actual requirements of alarm system testing in HanYi Railway, a total of five categories and 37 field test cases are carried out. The test cases include: [7]

(1) Function tests of Satellite differential base station: including differential data transmission and reception, home satellite differential base station switching;

(2) Basic functional tests: including identification of main line in non-parallel sections, pick-up on sidings in up/down line, departure on sidings in up/down line;

(3) Train operation scenario tests: including no alarm/prompt alarm/approaching alarm/warning alarm scenario when two trains are tracing on 
the same direction, pick-up and departure scenario when the back train is tracing the train ahead (adding and deleting the train ahead), two trains stopping scenario in the same station, the back train disposing scenario when the front train is stopping in railway section or main track in station, the scenario when two trains are running towards each other on the same track, the scenario when two trains are intersecting on main line, the scenario when the ground signal is updating in Train Control Center;

(4) Failure mode tests: including possible communication failure, positioning failure, failure of differential base station, and all kinds of recovery of failure;

(5) Supplemental tests: including the system performance under the failure of differential base station.

The test mainly aims at the following two focuses:

- Train positioning performance

- Alarm function implementation

Test results are mainly recorded in the form of field working history and working $\log$.

\section{Testing analysis}

According to three function indexes including the judgment of the train's position, analysis of train's running mode and its transforming, plus the state of alarming and its transforming, the test data have been analyzed and discussed.

\subsection{Analysis of positioning performance}

On-board positioning adopts differential positioning technology. The team respectively set satellite positioning differential base stations in Hanchuan station and Wujiashan line station on HanYi Railway, and set their jurisdictions based on the geographical distribution of the railway line. Fig. 8 shows the test data of

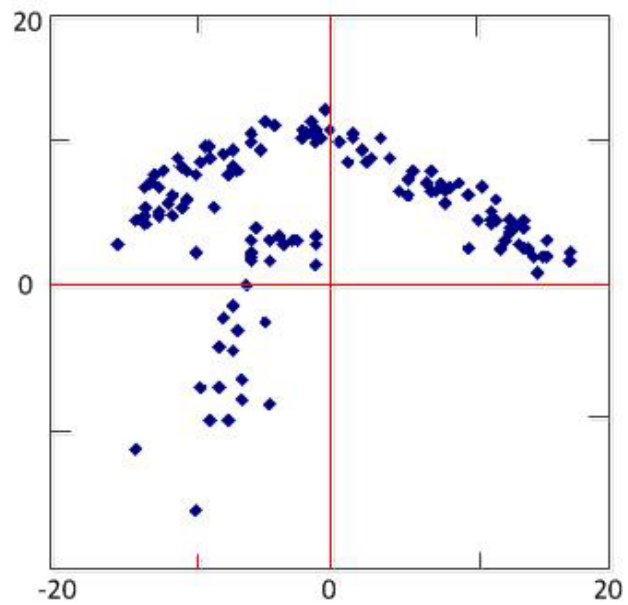

Figure 8: Accuracy of differential positioning (Unit: $\mathrm{cm}$ ). 
fix-point differential positioning collected at arrival signal in Tianmen South Station. Its average horizontal error is $21.432 \mathrm{~cm}$ and error variance is 34.792 [8].

By evaluating the data fusion and map-matching result and comparing the kilometer marker data of on-board computer and locomotive safety information monitoring device (TAX box) at the same time, the performance of mapmatching algorithm can be verified. Fig. 9 gives the error between 100 calculated and actual kilometer markers of main track in HanYi Railway. It can be seen that the train position error can be controlled within 8 meters, which meets the requirements of high-speed train tracing and an approach-alarming system.

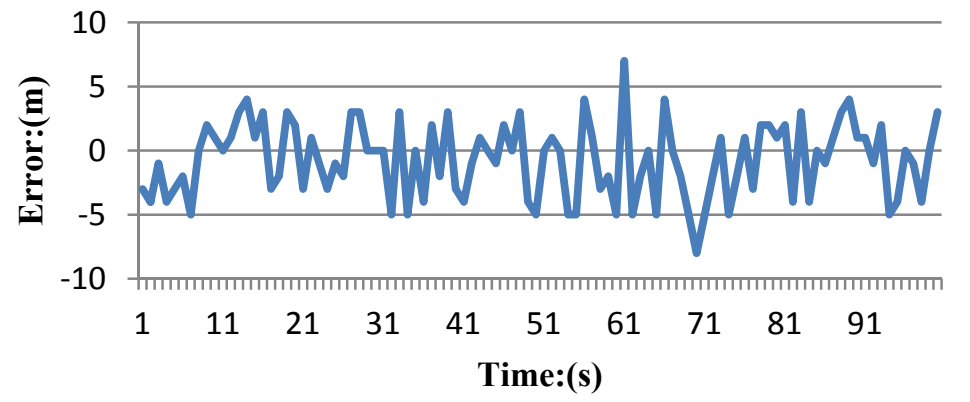

Figure 9: Performance test of data fusion and map-matching.

Fig. 10 presents the positioning performance in train station. The train stopped and departed at 5G of Hanchuan Station and its positioning result determines track occupancy accurately.
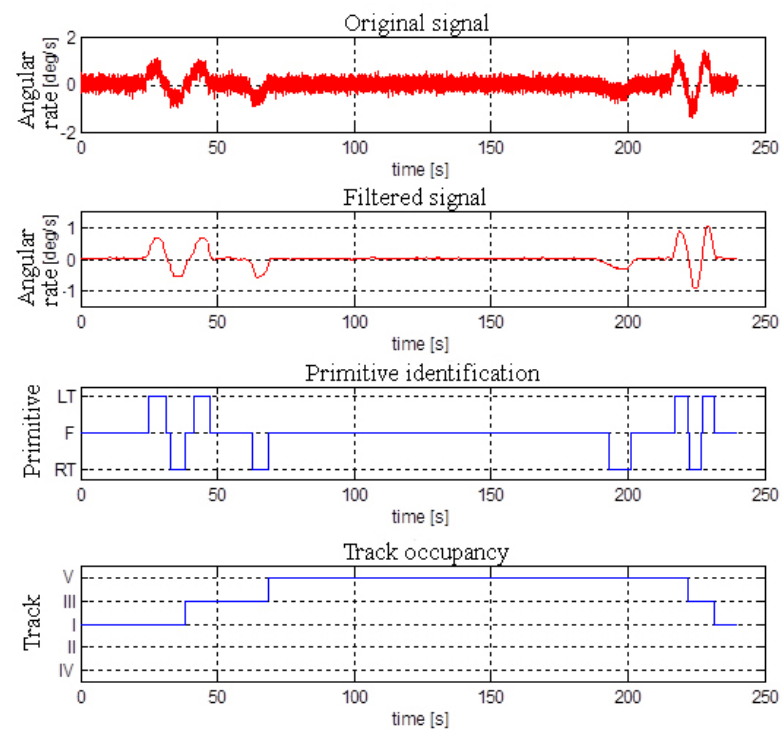

Figure 10: Determination of track occupancy at station. 


\subsection{Implementation of alarm function}

The test scenario when the ground signal in updating in Train Control Center is carried out in field testing. The team simulated ground signal failure of YongWen Railway to test the alarm system. It mainly tests the implementation of alarm distance and effective alarm performance under the condition of ground signal not functioning. The test case scenario is shown in Fig. 11. The alarm threshold is set as follows: when the distance is between 10 and $50 \mathrm{~km}$ from the train ahead, it prompts; when the distance is between 5 and $10 \mathrm{~km}$, it alarms; when the distance is less than $5 \mathrm{~km}$, it warns.

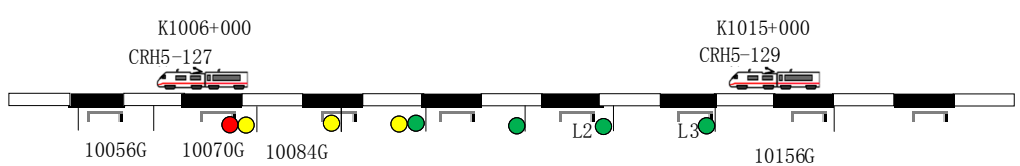

1. Two trains park at test points.

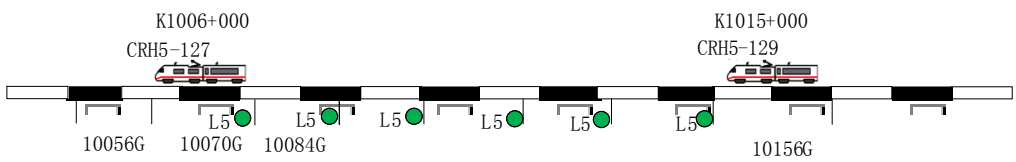

2. Remove red light strip protection of ground signal.

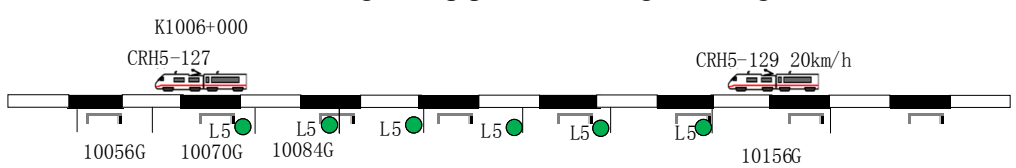

3. The back train runs at limited speed.

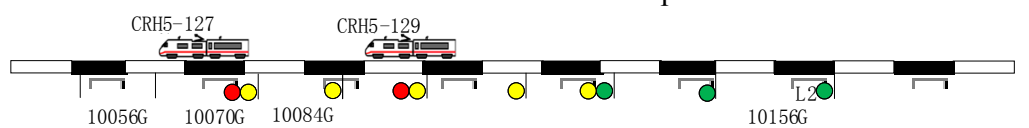

4. The back train stops and ground signal recovers.

Figure 11: Set of test case scenarios.

The final alarm results are shown in the following table:

Table 1: Alarm results.

\begin{tabular}{c|l|l|l|l|l|l}
\hline $\begin{array}{c}\text { Front train } \\
\text { position }\end{array}$ & $\begin{array}{l}\mathrm{K} 1006 \\
+000\end{array}$ & $\begin{array}{l}\mathrm{K} 1006 \\
+000\end{array}$ & $\begin{array}{l}\mathrm{K} 1006 \\
+000\end{array}$ & $\begin{array}{l}\mathrm{K} 1006 \\
+000\end{array}$ & $\begin{array}{l}\mathrm{K} 1003 \\
+012\end{array}$ & $\begin{array}{l}\mathrm{K} 998 \\
+024\end{array}$ \\
\hline $\begin{array}{c}\text { Back train } \\
\text { position }\end{array}$ & $\begin{array}{l}\mathrm{K} 1020 \\
+000\end{array}$ & $\begin{array}{l}\mathrm{K} 1016 \\
+007\end{array}$ & $\begin{array}{l}\mathrm{K} 1010 \\
+989\end{array}$ & $\begin{array}{l}\mathrm{K} 1008 \\
+500\end{array}$ & $\begin{array}{l}\mathrm{K} 1008 \\
+500\end{array}$ & $\begin{array}{l}\mathrm{K} 1008 \\
+500\end{array}$ \\
\hline $\begin{array}{c}\text { Back train } \\
\text { alarm }\end{array}$ & Prompt & $\begin{array}{l}\text { Alarm } \\
\text { (early } \\
\text { warning) }\end{array}$ & Warning & Warning & $\begin{array}{l}\text { Alarm } \\
\text { (early } \\
\text { warning) }\end{array}$ & Prompt \\
\hline
\end{tabular}

It can be concluded that under given alarm conditions, the alarm function can be fully implemented and can achieve the expected requirements. 


\section{Conclusion}

According to the application demand of high-speed train tracing and the approach-alarming system, Beijing Jiaotong University research group successfully conducts system testing for on-line operation.

On field testing on actual railway line of HanYi Railway, the system has been verified in current train operating environment and operating characteristics. And results show that under current conditions of installment, signal, power supply and operation mode (at $200 \mathrm{~km} / \mathrm{h}$ ), high-speed train tracing and an approachalarming system can fulfill all alarm functions in functional requirement.

The test results provide important support for further implementing system scheme, optimizing working conditions of system and producing equipments eventually. Also the system will play a critical role in the safety of high-speed train operation.

\section{References}

[1] Schneider U, Troelsen J., GPS based positive train control: Developments and field trials. Proc. of $7^{\text {th }}$ Int. Conf. On Computers in Railway, pp. 1271$1281,2000$.

[2] Hongdou Jin, Baigen Cai, Jiang Wang, et al., Design and Realization of Onboard device for High-speed train Tracing and Approach-Alarming System. Railway Signalling \& Communication, 49(5), pp. 73-77, 2013.

[3] Jiang Liu, Baigen Cai, Jian Wang, Precise Position Estimation of High Speed Railway Train for Collision Early Warning System. International Journal of Advancements in Computing Technology (IJACT), 5(8), pp. 1155-1166, 2013.

[4] Mueller K. T., Darren D., Field test results with a high speed rail prototype GPS locomotive location system. Proceedings of the $16^{\text {th }}$ International Technical Meeting of the Satellite Division of The Institute of Navigation (ION GPS/GNSS). Manassas, VA: Institute of Navigation, pp. 245-257, 2003.

[5] Baigen Cai, Xihui Yan, Jian Wang, et al., Automatic identification algorithm of train track occupancy. Journal of Traffic and Transportation Engineering, 10(6), pp. 111-115, 2010.

[6] Yuming Fan, Baigen Cai, Jian Wang, Wei Shangguan \& Yi An, Research on Beidou-Gyro integrated positioning based track occupancy discrimination method. Journal of the China Railway Society, 35(9), pp. 46-51, 2013.

[7] Test program for High-speed train Tracing and Approach-Alarming System; China Academy of Railway Sciences, Beijing Jiaotong University, et al.

[8] Qinlong Wang, Baigen Cai, Jian Wang, Wei Shangguan \& Jiang Liu, Design of GPS differential base station for train tracing and approachalarming system. Railway Signalling \& Communication, 49(4), pp. 61-65, 2013. 